Research article

\title{
A comparative genomics approach to understanding the biosynthesis of the sunscreen scytonemin in cyanobacteria Tanya Soule ${ }^{\dagger 1,2}$, Kendra Palmer ${ }^{\dagger 1}$, Qunjie Gao ${ }^{1}$, Ruth M Potrafka1, Valerie Stout ${ }^{1}$ and Ferran Garcia-Pichel ${ }^{* 1}$
}

\begin{abstract}
Address: ${ }^{1}$ School of Life Sciences, Arizona State University, Tempe, Arizona 85287, USA and ${ }^{2}$ Environmental Biotechnology, Savannah River National Laboratory, Aiken, South Carolina 29808, USA

Email: Tanya Soule - tanya.soule@srnl.doe.gov; Kendra Palmer - kendra.harris@asu.edu; Qunjie Gao - gao.qunjie@asu.edu; Ruth M Potrafka - ruth.potrafka@asu.edu; Valerie Stout - vstout@asu.edu; Ferran Garcia-Pichel* - ferran@asu.edu

* Corresponding author †Equal contributors
\end{abstract}

Published: 24 July 2009

BMC Genomics 2009, 10:336 doi:10.1186/147|-2164-10-336
Received: 6 January 2009

Accepted: 24 July 2009

This article is available from: http://www.biomedcentral.com/I47I-2/64/I0/336

(C) 2009 Soule et al; licensee BioMed Central Ltd.

This is an Open Access article distributed under the terms of the Creative Commons Attribution License (http://creativecommons.org/licenses/by/2.0), which permits unrestricted use, distribution, and reproduction in any medium, provided the original work is properly cited.

\begin{abstract}
Background: The extracellular sunscreen scytonemin is the most common and widespread indolealkaloid among cyanobacteria. Previous research using the cyanobacterium Nostoc punctiforme ATCC 29133 revealed a unique I8-gene cluster (NpRI276 to NpRI259 in the N. punctiforme genome) involved in the biosynthesis of scytonemin. We provide further genomic characterization of these genes in $\mathrm{N}$. punctiforme and extend it to homologous regions in other cyanobacteria.

Results: Six putative genes in the scytonemin gene cluster (NpRI276 to NpRI27I in the N. punctiforme genome), with no previously known protein function and annotated in this study as scyA to scyF, are likely involved in the assembly of scytonemin from central metabolites, based on genetic, biochemical, and sequence similarity evidence. Also in this cluster are redundant copies of genes encoding for aromatic amino acid biosynthetic enzymes. These can theoretically lead to tryptophan and the tyrosine precursor, p-hydroxyphenylpyruvate, (expected biosynthetic precursors of scytonemin) from end products of the shikimic acid pathway. Redundant copies of the genes coding for the key regulatory and rate-limiting enzymes of the shikimic acid pathway are found there as well. We identified four other cyanobacterial strains containing orthologues of all of these genes, three of them by database searches (Lyngbya PCC 8106, Anabaena PCC 7I20, and Nodularia CCY 94I4) and one by targeted sequencing (Chlorogloeopsis sp. strain Cgs-089; CCMEE 5094). Genomic comparisons revealed that most scytonemin-related genes were highly conserved among strains and that two additional conserved clusters, NpF5232 to NpF5236 and a putative two-component regulatory system ( NpFI 278 and $\mathrm{NpF}$ I277), are likely involved in scytonemin biosynthesis and regulation, respectively, on the basis of conservation and location. Since many of the protein product sequences for the newly described genes, including ScyD, ScyE, and ScyF, have export signal domains, while others have putative transmembrane domains, it can be inferred that scytonemin biosynthesis is compartmentalized within the cell. Basic structural monomer synthesis and initial condensation are most likely cytoplasmic, while later reactions are predicted to be periplasmic.
\end{abstract}

Conclusion: We show that scytonemin biosynthetic genes are highly conserved among evolutionarily diverse strains, likely include more genes than previously determined, and are predicted to involve compartmentalization of the biosynthetic pathway in the cell, an unusual trait for prokaryotes. 


\section{Background}

The sunscreen scytonemin (Figure 1A) is exclusively produced by some strains of cyanobacteria in response to UVA irradiation (315 to $400 \mathrm{~nm}$ wavelength). It is deposited as a yellow-brown pigment in the exopolysaccharide sheaths or capsules of the cyanobacteria which produce it [1]. Scytonemin can protect the organism by effectively minimizing damage associated with UVA exposure [2], usually associated with the photoproduction of singlet oxygen $[3,4]$, as well as the sensitization of endogenous photosensitizers such as flavins and heme groups [3]. In the natural environment, organisms capable of producing scytonemin are often under restricted growth and metabolism due to harsh environmental conditions, and are usually found on soil surfaces, rocks, and marine intertidal mats $[5,6]$. Scytonemin offers these organisms an alternative to traditional UVA repair methods by providing them with a passive, preventative mechanism to resist UVA irradiation before it ever reaches cellular targets.

The UV-absorbing ability of scytonemin is based on its chemical structure, a symmetrical indole-alkaloid consisting of fused heterocyclic units [7]. The biosynthesis of scytonemin likely involves tryptophan and tyrosine derivatives [8], both of which are known to absorb ambient UVB irradiation $[9,10]$. Although much is known about the biochemistry and ecology of scytonemin, very little was known until recently concerning its biosynthesis and molecular genetics.

In our previous study, using the model organism Nostoc punctiforme ATCC 29133 (N. punctiforme), we were able to characterize an 18-gene region associated with the biosynthesis of scytonemin [11], and compare that genomic

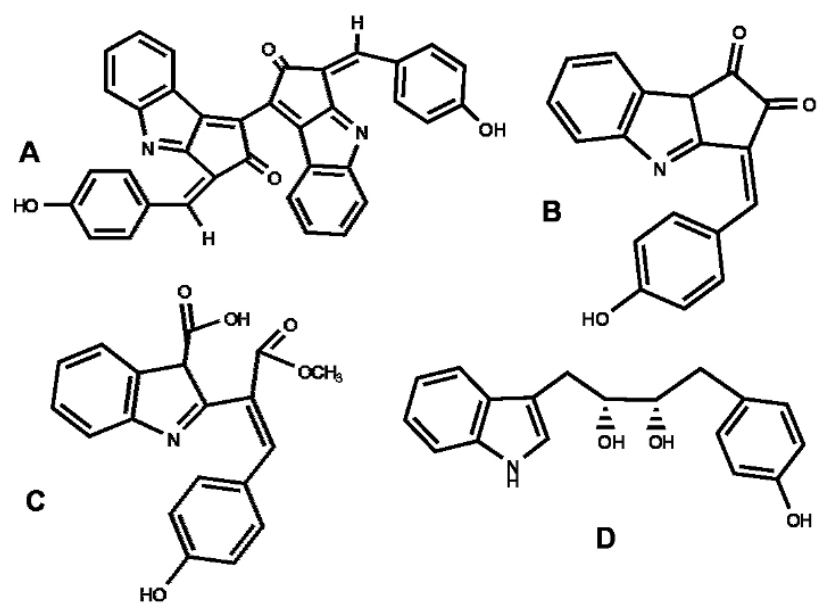

Figure I

Chemical structures of scytonemin and its precursors. (A) Scytonemin, (B) nostodione A, (C) prenostodione, and (D) diolmycin AI. region to a similar gene cluster in Anabaena PCC 7120 (Anabaena). Since then, two additional cyanobacterial genomes were sequenced, Lyngbya PCC 8106 (Lyngbya) and Nodularia spumigena CCY 9414 (Nodularia), which also contain orthologues to the scytonemin-associated genes from $N$. punctiforme [11], and the putative roles of the initial genes in scytonemin biosynthesis have been corroborated in a recent study [12]. Additionally, we were able to sequence several putative biosynthetic genes from this region in another scytonemin-producing cyanobacterium, Chlorogloeopsis sp. strain Cgs-089 [1]. Chlorogloeopsis can also be identified by the strain number CCMEE 5094, maintained by the Culture Collection of Microorganisms from Extreme Environments at the University of Oregon http://cultures.uoregon.edu/. Genomic comparisons of the scytonemin-associated genes from all five cyanobacteria above suggest many similarities and have resulted in the discovery of additional genes in $N$. punctiforme that may be associated with scytonemin biosynthesis and regulation. Here we describe and characterize genes that appear to be essential for scytonemin biosynthesis, and develop the first hypothetical model for the cellular compartmentalization of scytonemin biosynthesis.

\section{Results and discussion}

Analysis of the scytonemin biosynthesis genomic region in N. punctiforme

In our previous study we proposed that the open reading frames (referred to herein as genes) NpR1276 to NpR1259 in the N. punctiforme genome comprise a functional unit dedicated to scytonemin biosynthesis [11]. Within this 18-gene cluster, there appears to be a functional separation between the upstream genes and those in the downstream region (Figure 2). Although some of the genes in the upstream region had not been associated with any protein function, others had been preliminarily annotated. For example, NpR1276 is annotated in GenBank as an acetolactate synthase, which is a thiamine pyrophosphate (TPP)-requiring enzyme. Functionally, acetolactate synthase is able to condense two pyruvate molecules [13] and is almost always found as part of the valine and isoleucine biosynthesis ilvBN operon [14]. NpR1276, on the other hand, is not found anywhere near $i l v$-genes in the N. punctiforme genome. It does, however, contain domains specific for a TPP-requiring enzyme [15], and it has been shown to have a similarly condensing activity on phenol- and indole-pyruvate moieties [12]. This constitutes sufficient divergence to revisit the annotation and rename the gene scyA. The next gene in the cluster, NpR1275, was annotated as leucine dehydrogenase $(g d h A)$. Even though the protein sequence has the necessary domain for glutamate and leucine dehydrogenase, both of which are structurally related $\mathrm{NAD}^{+}$-dependent oxidoreductases [16], it only shares a $48 \%$ similarity to the leucine dehydrogenase characterized from Thermoac- 


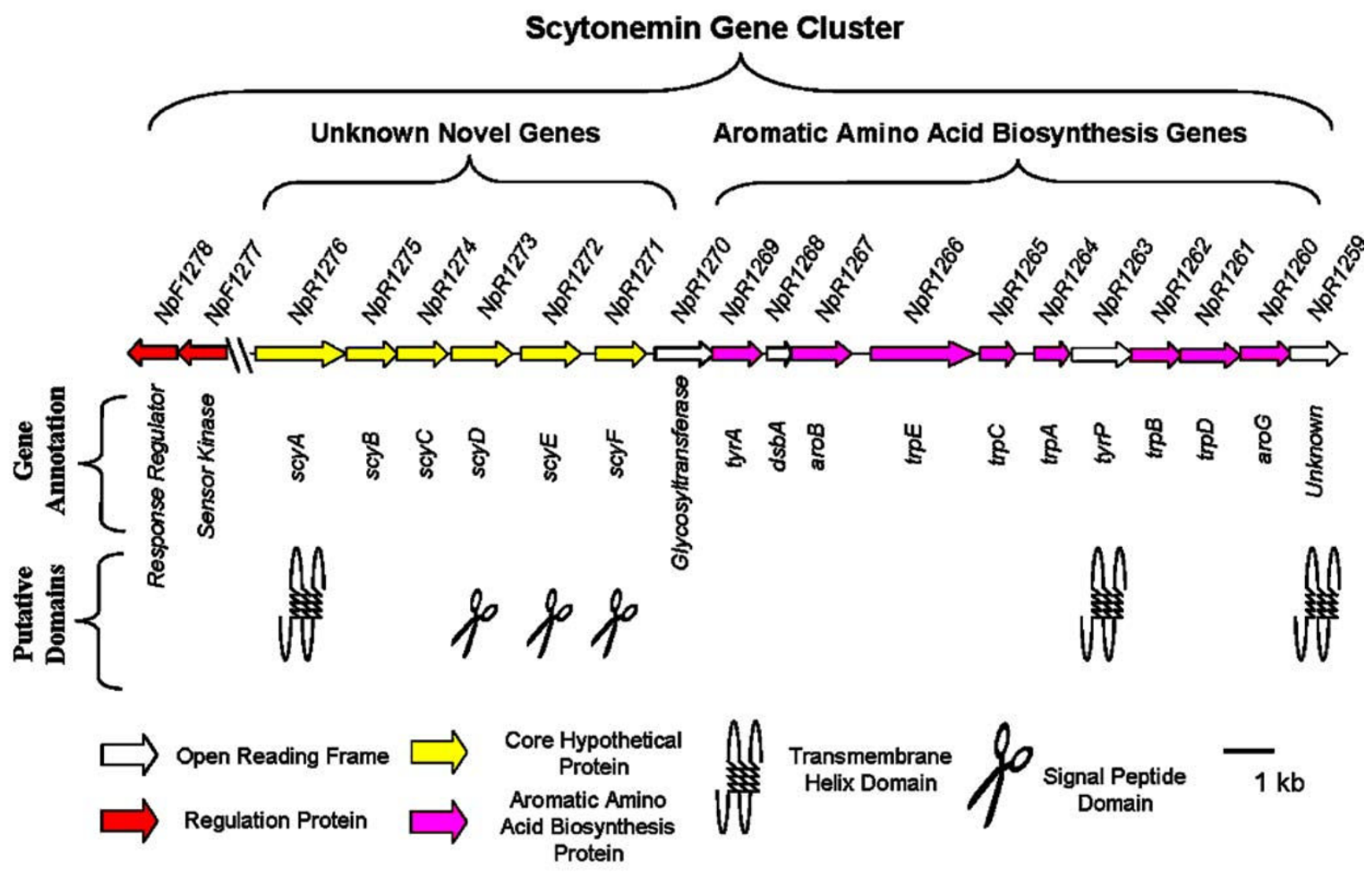

Figure 2

Genomic region in N. punctiforme associated with scytonemin biosynthesis. Arrows represent genes and their transcriptional orientation. All annotations are taken from the $N$. punctiforme genomic database and hash marks indicate a break in the distance scale.

tinomyces intermedius. The GdhA from T. intermedius is involved in catalyzing the oxidative deamination of branched amino acids [17]. The product of NpR1275 has a similar activity, but involves the oxidative deamination of aromatic amino acids [12]. As in the case of $s c y A$, there are sufficient differentiating traits to rename the gene as $s c y B$. Even though a protein function cannot be readily predicted for the next four genes (NpR1274 to NpR1271), NpR1273 has experimentally been shown to prevent scytonemin production when inactivated through transposon insertion [11]. For consistency, given the lack of alternatives, and in keeping with the continuity of $s c y A$ and $s c y B$, we propose that these four genes encode for truly unique proteins likely essential to scytonemin biosynthesis, and will be referred to as $s c y C-F$, respectively.

The predicted structural features found in some of these genes are also interesting and support a cellular compartmentalization of scytonemin biosynthesis. For example, ScyD, ScyE, and ScyF, none of which had been assigned a protein function by annotation, each contain a signal peptide export domain in their derived protein sequence. These N-terminal signature sequences are often associated with periplasmic proteins, suggesting that some stages of scytonemin biosynthesis may occur in the periplasm. Furthermore, the protein sequences of ScyA, TyrP, and NpR1259 all contain at least one transmembrane domain. The software program PSLpred [18], which predicts the subcellular localization of bacterial proteins based on their protein sequences, suggests that TyrP may also function on the periplasmic side, while ScyA and NpR1259 likely function on the cytoplasmic side. While the protein sequence of NpR1268 does not have an N-terminal export domain, the fact that it resembles $d s b A$, a dithiol-disulfide isomerase (oxidoreductase) that facilitates the formation of disulfide bridges in the folding of periplasmic proteins [19], suggests that it may also localize to the periplasm. This leads us to speculate that a dithiol-disulfide isomerase of this kind could be important as an accessory to the other proteins predicted to be active in the periplasm. Thus, the upstream region of the cluster is comprised of novel genes likely involved directly in the assembly of scytonemin biosynthesis, where early condensing reactions occur in the cytoplasm and presumably later steps appear to be localized to the periplasm. 
Most of the genes located towards the downstream portion of the cluster are clearly associated by similarity with the biosynthesis of aromatic amino acids $[11,20]$. Furthermore, they do not contain structural motives that predict their association with cellular membranes or their transport to the periplasmic space. In this region of the cluster are genes predicted to code for the first two enzymes of the shikimic acid pathway (aroG, aroB), leading to the formation of 5-dehydroquinate. All of the genes necessary for the biosynthesis of tryptophan from chorismate (trpE, $\operatorname{trp} C, \operatorname{trp} A, \operatorname{trp} B, \operatorname{trp} D)$ are also present, while only prephenate dehydrogenase (encoded by tyr $A$ ) is present from the tyrosine biosynthesis pathway, thus ending that pathway at $p$-hydroxyphenylpyruvate, one amination short of tyrosine $[21,22]$. In fact, on the basis of chemical structures [7], p-hydroxyphenylpyruvate is a theoretically more direct precursor for scytonemin than tyrosine.

One of the most significant observations regarding these aromatic amino acid genes is that there is at least one other copy of each of them elsewhere in the genome of $N$. punctiforme at dispersed loci. Genes in this dispersed set find homologues in all other cyanobacteria sequenced so far and thus likely have a housekeeping function [20]. The cluster of redundant copies of aromatic amino acid biosynthetic genes, by contrast, appears to be unique and always spatially associated with the scytonemin cluster in the few cyanobacterial genomes that have it. Therefore, it is reasonable to hypothesize that the downstream region of the scytonemin cluster is likely dedicated to supplying the building blocks for the biosynthesis of scytonemin, while the standard housekeeping copies remain important for central metabolism. This is supported by the differential up-regulation of these redundant genes along with the induction of scytonemin synthesis in N. punctiforme, while the expression levels of the housekeeping genes remain unaltered [23].

Two genes in the downstream region of the cluster have previously been assigned putative protein functions not related to aromatic amino acid biosynthesis. NpR1270 shows similarity to a putative glycosyltransferase, with $77 \%$ identity to a glycosyltransferase in Nodularia. Interestingly, some glycosyltransferases in bacteria have been linked to exopolysaccharide biosynthesis [24]. Specifically, in Nostoc commune, the synthesis of scytonemin is coupled to the synthesis of the exopolysaccharide [25]. The protein sequence of NpR1263 has a transmembrane domain and is annotated as a putative tyrosinase, TyrP, a copper monooxygenase that can hydroxylate monophenols and oxidize o-diphenols to o-quinols [26]. Indeed, NpR1263 has the essential conserved residues for $\mathrm{Cu}^{2+}$ binding and is a putative tyrosinase-like protein. It is unique, in that it does not have any cyanobacterial protein sequence homologs in GenBank, and it can be predicted to play an important role in scytonemin biosynthesis, as explained below. The other downstream gene is NpR1259, the last gene in this cluster. It has two putative transmembrane domains and was annotated as a hypothetical membrane protein, since it lacks real homologies with known genes.

Upstream from the gene cluster are two genes that might be involved in the regulation of scytonemin biosynthesis, given their high degree of conservation in sequence and location among distantly related strains (see below). These protein sequences reveal strong similarities to twocomponent signal transduction systems. These systems typically involve the autophosphorylation of a histidine kinase (in our case, NpF1277) and the subsequent transfer of the phosphate group to an aspartate on the protein. This phosphorylated aspartate then acts as a phosphodonor to a response regulator protein (in our case, NpF1278), which ultimately turns on the transcription of the genes the system regulates $[27,28]$. NpF1277 likely belongs to class II histidine kinases, which are characterized by the presence of PAS/PAC sensory domains that are generally sensitive to oxygen, redox, or light [29]. $\mathrm{NpF} 1278$ is a class II response regulator (RR) [30] predicted to be a positive transcriptional regulator [31]. A working hypothesis is that NpF1277 and NpF1278 might regulate the adjacent genomic region (NpR1276 to NpR1259) associated with scytonemin biosynthesis.

\section{Comparative genomics of the scytonemin gene cluster}

The scytonemin-associated gene region was identified in three additional strains, belonging to the genera Anabaena, Lyngbya, and Nodularia, among all bacteria whose genomes have been completely sequenced. Genomic arrangements of homologous genes were similar to those of $N$. punctiforme (Figure 3A). The scytonemin core genes (scyA-F) are conserved in all four genomes, their orthologs are at least $42 \%$, and most greater than $65 \%$, similar to one another (Table 1), and they are positioned near sets of redundant copies of aromatic amino acid biosynthesis genes. These redundant copies are orthologous to the exact same set found in the $N$. punctiforme genomic region. The only other gene in the cluster conserved across all four genomes was the response regulator, NpF1278 in N. punctiforme.

In the scytonemin gene cluster of Anabaena, Lyngbya, and Nodularia, there are five conserved genes downstream of $s c y F$ that are absent from the $N$. punctiforme cluster (shown in black in Figure 3A). In hindsight inspection, orthologs of these genes could readily be identified elsewhere on $N$. punctiforme's chromosome. There, they comprised a fivegene satellite cluster with all five genes oriented in the same transcriptional direction (NpF5232 to NpF5236). In N. punctiforme, NpF5232 and NpF5235 are annotated as unknown hypothetical proteins, while NpF5233, NpF5234, and NpF5236 are annotated as a putative 

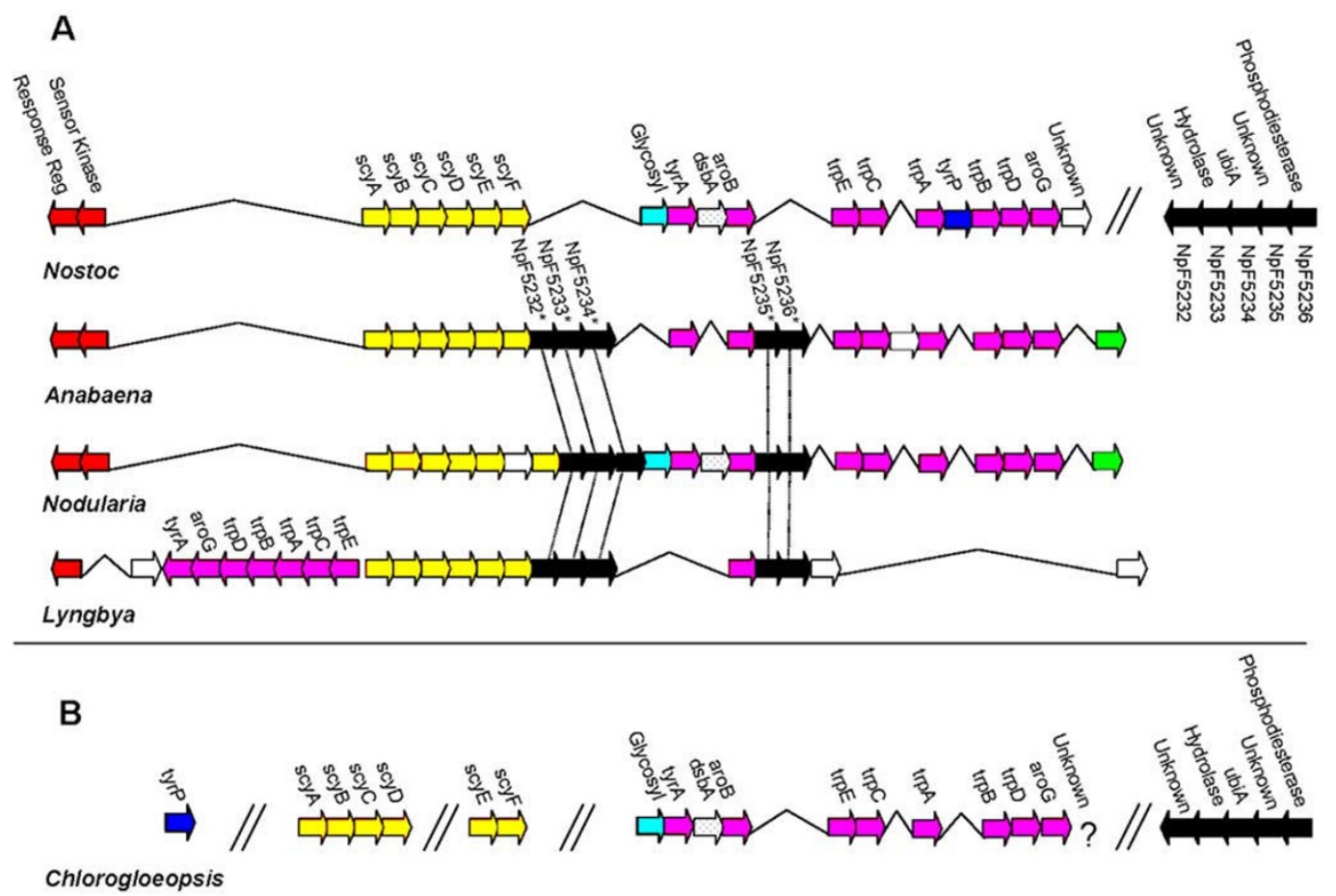

\section{Figure 3}

Genomic region associated with scytonemin biosynthesis in several strains of cyanobacteria. (A) Genomic region in N. punctiforme, Anabaena, Nodularia, and Lyngbya (not drawn to scale). Arrows represent the transcriptional orientation of the genes, which are filled in according to the functional category as follows: red, regulatory proteins; yellow, scytonemin core genes; pink, aromatic amino acid biosynthetic genes; black, orthologs to the five-gene satellite cluster in N. punctiforme; white, genes without homologues among the strains studied; all other colors represent orthologs. Hash marks delimit the two gene clusters in $N$. punctiforme and carats connect adjacent genes. The vertical alignments of the genes facilitate the visual representation of orthologous proteins, with one exception; the white gene positioned in the Nodularia yellow gene cluster causes a shift in the vertical alignment of the corresponding orthologous genes which is corrected at the position of the "glycosyltransferase". Orthologues to the five-gene satellite cluster from N. punctiforme are specified with a star and dashed lines facilitate their alignment. (B) Genomic region associated with scytonemin biosynthesis of Chlorogloeopsis, vertically aligned to match $N$. punctiforme in (A). An ortholog to the last gene in the N. punctiforme cluster has not been identified in Chlorogloeopsis and tyrP does not appear to be integrated within the gene cluster, although it is present in the genome. Genes that are not continuously linked are shown by the insertion of hash marks.

metal-dependent hydrolase, prenyltransferase (ubiA), and type I phosphodiesterase, respectively. However, these annotations are based on weak similarity, and the orthologs of each of these genes are annotated as unknown hypothetical proteins in the Anabaena, Lyngbya, and Nodularia genomes. At this point, it seems that ambiguity calls for a cautious approach by postponing a specific annotation for these genes.

In a previous study we determined that Anabaena was unable to produce scytonemin [11], even though it contained many of the genes in the scytonemin cluster, and interpreted this as a case of relic genetic information. It was thus important to test if scytonemin was produced in the other strains used in the comparisons. We could elicit the production of scytonemin neither in Lyngbya nor in Nodularia, upon exposing cultures of each strain to UVA radi- ation, which is the standard procedure to achieve biosynthetic induction (see Methods). It is possible that these strains may have had the ability to produce scytonemin at some point in their evolutionary history, but have now lost it, since laboratory strains are rarely, if ever, exposed to the doses of UVA required for scytonemin biosynthesis. Furthermore, since scytonemin is a passive sunscreen it is most effective in environments with pulsed resource availability as explained above. Since Anabaena and Nodularia are planktonic [32], their need for a passive sunscreen is not as crucial as it is for the Nostoc and Chlorogloeopsis strains of terrestrial habitats [32]. Although some strains of Lyngbya produce scytonemin, Lyngbya PCC 8106 does not produce it. This may be because the marine inter-tidal zone that it was isolated from had varying degrees of resource availability and UV exposure, thus this Lyngbya strain may have not needed a passive sunscreen. 
Table I: Cyanobacterial orthologs to the scytonemin-associated genes of $N$. punctiforme.

\begin{tabular}{|c|c|c|c|c|}
\hline Gene in Nostoc & Description in Nostoc & $\%$ Identity ${ }^{a}$ to Anabaena & $\%$ Identity to Nodularia & \% Identity to Lyngbya \\
\hline $\mathrm{NpF} / 278$ & response regulator & 67 & 71 & 47 \\
\hline $\mathrm{NpF} / 277$ & sensor kinase & 68 & 72 & 39 \\
\hline NpRI 276 & scyA (ilvB) & 74 & 79 & 60 \\
\hline $\mathrm{NpR} / 275$ & $\operatorname{scyB}(g d h A)$ & 79 & 81 & 64 \\
\hline NpRI274 & scyc & 82 & 81 & 71 \\
\hline NpRI273 & scyD & 71 & 66 & 42 \\
\hline NpRI272 & scyE & 47 & 65 & 42 \\
\hline NpRI27I & scyF & 55 & 52 & 63 \\
\hline $\mathrm{NpR} / 270$ & glycosyltransferase & 75 & 77 & 62 \\
\hline NpRI269 & tyrA & 72 & 74 & 41 \\
\hline NpRI268 & frnE & $\mathrm{N} / \mathrm{A}^{b}$ & 69 & $N / A$ \\
\hline $\mathrm{NpR} / 267$ & $\operatorname{aro} B$ & 71 & 78 & 64 \\
\hline NpRI266 & $\operatorname{trp} E$ & 81 & 81 & 65 \\
\hline NpRI265 & $\operatorname{trpC}$ & 79 & 80 & 60 \\
\hline NpRI 264 & $\operatorname{trpA}$ & 85 & 81 & 70 \\
\hline NpRI263 & tyrP & $N / A$ & $N / A$ & $N / A$ \\
\hline $\mathrm{NpR} / 262$ & $\operatorname{trp} B$ & 88 & 88 & 85 \\
\hline NpRI26I & $\operatorname{trpD}$ & 81 & 81 & 65 \\
\hline $\mathrm{NpR} / 260$ & aroG & 84 & 86 & 74 \\
\hline NpRI 259 & unknown & $\mathrm{N} / \mathrm{A}$ & N/A & N/A \\
\hline NpF5232 & unknown & 57 & 58 & 52 \\
\hline NpF5233 & hydrolase & 78 & 80 & 81 \\
\hline NpF5234 & ubiA & 64 & 64 & 49 \\
\hline NpF5235 & unknown & 64 & 64 & 59 \\
\hline NpF5236 & phosphodiesterase & 71 & 74 & 67 \\
\hline
\end{tabular}

$a$ Data are based on amino acid sequences

$b$ Similar sequences are not in the corresponding genome

Given these results, it seemed important to obtain sequences for the scytonemin-associated region from another scytonemin-producing strain besides $N$. punctiforme. Chlorogloeopsis sp. strain Cgs-O-89 [1], a cyanobacterium known to produce scytonemin [2], was selected for this purpose. Using targeted PCR based on primers designed from the N. punctiforme genome, we were able to amplify and sequence several genes from the genomic region associated with scytonemin biosynthesis of Chlorogloeopsis, and found that their genomic arrangement was very similar to that of $N$. punctiforme (Figure $3 \mathrm{~B}$ ). Additionally, the five-gene satellite cluster from $N$. punctiforme was found and sequenced in Chlorogloeopsis as a continuous segment. As in N. punctiforme, the Chlorogloeopsis satellite gene cluster was not continuous with the scytonemin-associated gene cluster. Although we were unable to link all of the scytonemin-associated gene orthologs of Chlorogloeopsis into a single contig, we could establish clear similarities between the Chlorogloeopsis and N. punctiforme gene clusters (Figure 3).

\section{Insights into the biosynthetic pathway and working model} for scytonemin biosynthesis

Scytonemin is a symmetrical dimeric molecule, and it is expected that each monomer is synthesized separately before condensing to form the dimer. In theory, if tryp- tophan and tyrosine were used as building blocks, the biosynthesis of scytonemin could involve as little as four to six biosynthetic steps. In fact, structural, genetic, and preliminary radiotracer evidence indicates that the biosynthesis of scytonemin starts from aromatic amino acid (or related) precursors $[7,8,11]$. Previously isolated natural products, with structural similarities to putative scytonemin subunits, also provide useful biosynthetic clues. Nostodione A (Figure 1B) has not only been isolated by ozonolysis of scytonemin [7], but has also been isolated from Nostoc commune and Scytonema hofmanni [33], two typical scytonemin-producing strains. It is thus logical to assume that nostodione $\mathrm{A}$ is the most likely monomeric intermediate of scytonemin. Prenostodione (Figure 1C), the methylated carboxylic acid precursor of nostodione A, has been reported from Nostoc sp. TAU strain IL-235, further suggesting that the origin of the biosynthetic pathway of scytonemin is from a condensation of tryptophan and phenylpropanoid derived subunits [34]. Indeed, a recent study found that deaminated tryptophan and tyrosine (indole-3-pyruvic acid and p-hydroxyphenylpyruvate, respectively) condense, through the action of ScyA and ScyB, to form an intermediate that is structurally similar to diolmycin A1 (Figure 1D) [12]. Diolmycin A1 has been isolated from Streptomyces sp. [35] and is a plausible intermediate in the scytonemin biosynthetic pathway. Further- 
more, oxidation of the tyrosine moiety appears to be essential for the biosynthesis of nostodione $A$, an essential precursor to scytonemin as mentioned above. We propose that this oxidation could be carried by the tyrosinase-like TyrP encoded for in the scytonemin gene cluster, since tyrosinases are known to promote monooxygenation in similar moieties [26]. It is interesting to note that the only scytonemin-associated gene in common between N. punctiforme and Chlorogloeopsis (the two proven scytonemin producers), that is absent from the other three strains (which, in our hands, do not produce it), is tyrP (putative tyrosinase). In fact, the gene appears to be absent from the genomes of these Lyngbya, Anabaena, and Nodularia strains altogether, as is the case for all other fully sequenced cyanobacterial genomes. We do note, however, that while the genome of Anabaena is complete, the Lyngbya and Nodularia genome projects are almost complete, and because of this we cannot determine with absolute certainty at the time of this publication if tyrP is absent from these genomes.

A working model of the subcellular compartmentalization of scytonemin biosynthesis in the cell, based on the above genomic analyses, is provided in Figure 4. Following a UVA radiation cue, the redundant genomic copies of the trp and tyr genes are expressed to lead the production of the tryptophan and p-hydroxyphenylpyruvate mono- mers from chorismate. The production of chorismate from central metabolites is boosted by additional expression of the genes aro $G$ and $a r o B$, which code for the regulatory and rate-limiting enzymes in the shikimic acid pathway, respectively. These precursors are first processed by ScyA, ScyB, ScyC, and NpR1259 in the cytoplasm. The resulting intermediaries are then excreted to the periplasm via some unknown membrane transport mechanism, as no known mechanism is coded for within the scytonemin cluster. There, they are subject to reactions orchestrated by the periplasmic enzymes ScyD, ScyE, ScyF, DsbA, and TyrP to produce the reduced form of scytonemin. Once secreted to the extracellular matrix, it auto-oxidizes and takes on its final yellow-brown appearance. Parallel studies suggest that a type IV secretion system, similar in mechanism to a bacterial conjugation system [36], is used to secrete scytonemin to the extracellular matrix (Soule et al., unpublished data). Once scytonemin is in the extracellular slime layer in sufficient quantities, it blocks the incoming UVA cue, thus returning the gene expression to background levels and halting the further synthesis of the sunscreen.

\section{Conclusion}

The conservation of genes and genomic arrangements between the $N$. punctiforme scytonemin biosynthesis gene cluster and the Chlorogloeopsis gene cluster allows us to

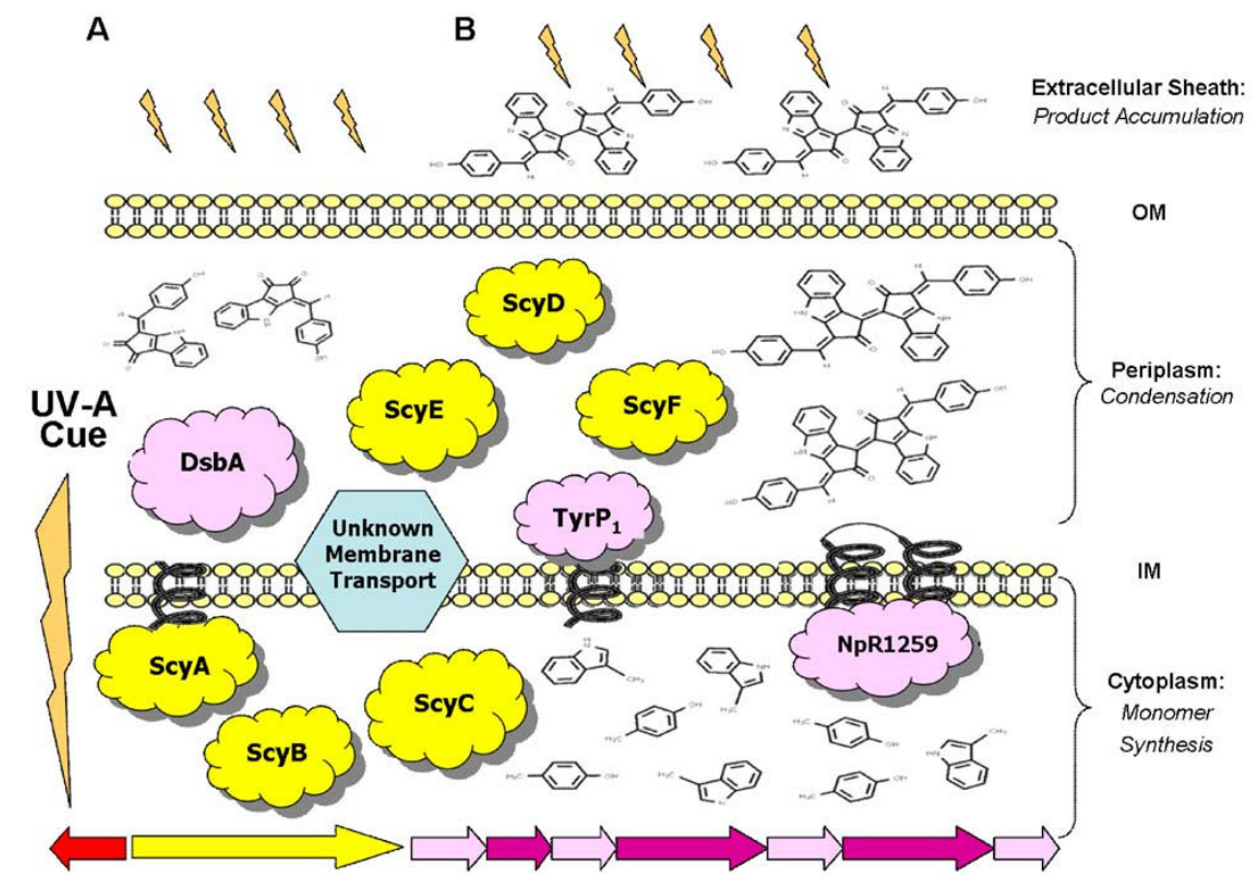

Figure 4

Working model of scytonemin biosynthesis based on genomic analyses. (A) UVA is absorbed and activates the proposed gene cluster to produce the corresponding protein products localized according to putative protein domains, see text for details. (B) UVA is blocked by scytonemin accumulated in the cyanobacterial sheath, which ultimately deactivates the transcription of the gene cluster and eliminates the need for the putative protein products. 
predict which genes are important in the biosynthesis of scytonemin. Since $s c y A$ to $s c y F$ are conserved across all of the strains described above, and are either unknown in function or putatively assigned a function, we expect that these six genes will provide the most useful information for determining the scytonemin biosynthetic pathway. Additionally, we have reason to associate the N. punctiforme genes NpF5232 to NpF5236 with the biosynthesis of scytonemin, and it is likely that the response regulator (NpF1278) and sensor kinase (NpF1277) upstream from the cluster are involved in regulating this system.

Furthermore, protein sequence data from several of the genes in the cluster provide us with clues regarding scytonemin biosynthesis and localization. While the roles of ScyA and ScyB in the preliminary stages of scytonemin biosynthesis are predicted to occur in the cytoplasm, a working model of scytonemin biosynthesis suggests periplasmic compartmentalization of the later biosynthetic stages. Overall, our analyses have increased our understanding of scytonemin biosynthesis and will facilitate the construction of more direct and efficient hypotheses for future experiments. Furthermore, as scytonemin has been documented as having anti-inflammatory [37] and antiproliferative properties [38], our work also helps those working on the biomedical potential of scytonemin and related compounds. This study constitutes a step forward in understanding the biosynthesis of secondary metabolites in bacteria and contributes a novel example of a biosynthetic pathway for a microbial indole-alkaloid. We hope that our contributions to understanding secondary metabolite biosynthesis in cyanobacteria will ultimately lead to the discovery of additional natural products and the pathways by which they are synthesized.

\section{Methods}

\section{Strains and cultivation}

Axenic stock cultures of each strain were maintained on plates solidified with $1.5 \%$ Noble agar. N. punctiforme was grown in Allen and Arnon medium (AA) [39] prepared at full strength for solid media or diluted four-fold for liquid media (AA/4). Anabaena, Nodularia, and Chlorogloeopsis cultures were grown in BG-11 [40], while Lyngbya was grown in a 1:1 mixture of BG-11 and ASN-III [41] supplemented with $10 \mu \mathrm{g} \mathrm{L}^{-1}$ vitamin $\mathrm{B}_{12}$. Cultures were grown in sterile flasks, under constant white light, at an intensity of $7 \mathrm{~W} \mathrm{~m}^{-2}$ provided by cool-white fluorescent tubes (General Electric), while shaking at $25^{\circ} \mathrm{C}$.

\section{Identification of strains with scytonemin-associated genes and subsequent phenotypic analysis}

Amino acid sequences of each protein involved in the biosynthesis of scytonemin from $N$. punctiforme was used in a BLASTp analysis in order to find orthologs in GenBank. Orthologous genes were mapped to establish their arrangement in the genomes of the strains harboring them. To determine whether or not these strains were capable of producing scytonemin, cultures were grown from stocks in liquid cultures [1] and acclimated to white light only $\left(10 \mathrm{~W} \mathrm{~m}^{-2}\right)$ for three days, followed by exposure to white light supplemented with UVA for five continuous days. The UVA was provided by $20-\mathrm{W}$ black-light fluorescent tubes (General Electric) at an intensity of $10 \mathrm{~W} \mathrm{~m}^{-2}$ with a spectral output of $365 \mathrm{~nm}$, as previously determined [2]. In some cases, the UVA intensity was gradually increased over the course of several days to acclimate more sensitive strains to $10 \mathrm{~W} \mathrm{~m}^{-2}$ of UVA. Additionally, a control culture for each strain was set under white light only. Following UVA exposure, the cells were harvested and the lipid-soluble pigments were extracted from whole cells in acetone. Extracts were analyzed on a commercial spectrophotometer for absorption from $350 \mathrm{~nm}$ to 750 $\mathrm{nm}$, a strong absorption peak at $384 \mathrm{~nm}$ indicated scytonemin had accumulated in the cells. Cultures were also observed microscopically for changes in extracellular pigmentation [1].

\section{Sequencing of scytonemin-associated genes in Chlorogloeopsis}

Total DNA was extracted from cultures of Chlorogloeopsis using a PCI (phenol; chloroform; isoamyl alcohol) extraction protocol [42]. Presence of DNA in the extracts was confirmed on ethidium bromide-stained 1\% agarose gels and quantified with a Nanodrop spectrophotometer (Thermo Fisher Scientific). The DNA was used as template for PCR with primers based on N. punctiforme sequences that were designed to bridge adjacent genes in the cluster. This approach was taken in order to capture the sequences of the corresponding genes and their flanking non-coding regions in Chlorogloeopsis. For PCR, 20 ng of Chlorogloeopsis DNA was used in $50 \mu \mathrm{L}$ reactions consisting of $1 \mu \mathrm{M}$ of each specific primer, $5 \mu \mathrm{L} 10 \times E x$ Taq DNA polymerase buffer, $4 \mu \mathrm{L}$ dNTP mixture ( $2.5 \mathrm{mM}$ each), and 1.25 units Ex Taq DNA polymerase (all from Takara Bio Inc.). $N$. punctiforme genomic DNA was the positive control while the negative control had no template DNA. PCR was done in a Bio-Rad iCycler Thermal Cycler with the following parameters: $95^{\circ} \mathrm{C}$ for 5 min then 35 cycles of $95^{\circ} \mathrm{C}$ for 1 min, $55^{\circ} \mathrm{C}$ for $1 \mathrm{~min}$, and $72^{\circ} \mathrm{C}$ for $1 \mathrm{~min}$, followed by an extension at $72^{\circ} \mathrm{C}$ for $10 \mathrm{~min}$. Products were confirmed on $1 \%$ agarose gels and the band of the expected size for each sample was excised using a sterile scalpel. The PCR products were purified using the QIAquick Gel Extraction Kit (Qiagen Sample and Assay Technologies) and sequenced commercially (Applied Biosystems). Sequences were used in a BLASTn analysis against the $N$. punctiforme genomic database http://www.jgi.doe.gov to verify that the correct region had been amplified. Gene sequences were used to construct the genomic arrangement of the scytonemin gene cluster in Chlorogloeopsis. 
Nucleotide sequences were submitted to GenBank under accession numbers FJ601359 to FJ601364 and FJ605302 to $\underline{\mathrm{F} 605317}$.

\section{Authors' contributions}

The concept for this study was provided by TS, FGP, and VS. Gene analyses and the working model for scytonemin biosynthesis was developed by TS, Chlorogloeopsis sequences and UV experiments were done by KP, cultures were provided from and maintained by RMP, and the pathway was analyzed by QG. Manuscript was written by TS with editorial help by VS and FGP. All authors have read and approved the final manuscript.

\section{Notes in proof}

While the manuscript was in review the genome sequences of Cyanothece sp. strains PCC 7424 and PCC 7822 became available to the public. Both of these strains contain the scytonemin genomic region in an arrangement similar to that found in Lyngbya PCC 8106. The ability of either of these strains to produce scytonemin has not been determined.

\section{Acknowledgements}

We would like to thank Scott Bingham and the Arizona State University DNA Laboratory staff for their assistance.

\section{References}

I. Garcia-Pichel F, Castenholz RW: Characterization and biological implications of scytonemin, a cyanobacterial sheath pigment. J Phycol 1991, 27:395-409.

2. Garcia-Pichel F, Sherry ND, Castenholz RW: Evidence for an ultraviolet sunscreen role of the extracellular pigment scytonemin in the terrestrial cyanobacterium Chlorogloeopsis sp. Photochem Photobiol 1992, 56(I): 17-23.

3. Jagger J: Solar-UV Actions on Living Cells. New York: Praeger; 1985.

4. Castenholz RW, Garcia-Pichel F: Cyanobacterial responses to UV-radiation. In The Ecology of Cyanobacteria Edited by: Whitton BA. Dordrecht, The Netherlands: Kluwer Academic Publishers; 2000:59I-6II.

5. Garcia-Pichel F, Belnap J: Microenvironments and microscale productivity of cyanobacterial desert crusts. J Phycol 1996, 32:774-782.

6. Rothrock M, Garcia-Pichel F: Microbial diversity of benthic mats along a tidal desiccation gradient. Environ Microbiol 2005, 7:593-60I.

7. Proteau P, Gerwick W, Garcia-Pichel F, Castenholz RW: The structure of scytonemin, an ultraviolet sunscreen pigment from the sheaths of cyanobacteria. Experientia 1993, 49:825-829.

8. Mandalka $A$ : Studies on scytonemin synthesis in cyanobacteria. Bremen, Germany: University of Bremen; 1999.

9. Strickland EH, Horwitz J, Billups C: Near-ultraviolet absorption bands of tryptophan, studies using indole and 3-methylindole as models. Biochemistry 1970, 9(25):49|4-4921.

10. Chen R: Measurements of absolute values in biochemical fluorescence spectroscopy. J Res Natl Bureau Standards 1972, 76A(6):593-606.

II. Soule T, Stout V, Swingley WD, Meeks JC, Garcia-Pichel F: Molecular genetics and genomic analysis of scytonemin biosynthesis in Nostoc punctiforme ATCC 29133. J Bacteriol 2007, I 89( I 2):4465-4472.

12. Balskus EP, Walsh CT: Investigating the initial steps in the biosynthesis of cyanobacterial sunscreen scytonemin. J Am Chem Soc 2008, I30(46): 15260-I I526I.
13. Chipman D, Baraka Z, Schloss JV: Biosynthesis of 2-aceto-2hydroxy acids: acetolactate synthases and acetohydroxyacid synthases. Biochem Biophys Acta 1998, I 385(2):40 I-4I9.

14. Umbarger HE: Biosynthesis of branched-chain amino acids. In Escherichia coli and Salmonella: Cellular and Molecular Biology Volume I. 2nd edition. Edited by: Neidhardt FC. Washington D.C. ASM Press; 1996:442-457.

15. Arjunan P, Umland T, Dyda F, Swaminathan S, Furey W, Sax M, Farrenkopf $B$, Gao Y, Zhang D, Jordan F: Crystal structure of the thiamin diphosphate-dependent enzyme pyruvate decarboxylase from the yeast Saccharomyces cerevisiae at 2.3 Å resolution. J Mol Biol 1996, 256:590-600.

16. Sanwal BD, Zink MW: L-Leucine dehydrogenase of Bacillus cereus. Arch Biochem Biophys 1961, 94:430-435.

17. Ohshima T, Nishida N, Bakthavatsalam S, Kataoka K, Takada H, Yoshimura T, Esaki N, Soda K: The purification, cloning, and sequencing of the gene for a halostable and thermostable leucine dehydrogenase from Thermoactinomyces intermedius. EurJ Biochem 1994, 222:305-312.

18. Bhasin M, Garg A, Raghara GPS: PSLpred: prediction of subcellular localization of bacterial proteins. Bioinformatics 2005, $21(10): 2422-2524$.

19. Noiva R: Enzymatic catalysis of disulfide formation. Protein Expr Purif 1994, 5(I): I-I3.

20. Xie G, Bonner CA, Brettin T, Gottardo R, Keyhani NO, Jensen RA: Lateral gene transfer and ancient paralogy of operons containing redundant copies of tryptophan-pathway genes in Xylella species and in heterocystous cyanobacteria. Genome Biol 2003, 4:RI4.

21. Hall GC, Flick MB, Gherna RL, Jensen RA: Biochemical diversity for biosynthesis of aromatic amino acids among cyanobacteria. J Bacteriol 1982, I49(I):65-78.

22. Herrmann KM, Somerville RL: Amino Acids: Biosynthesis and Gene Regulation. London: Addison-Wesley Publishing Company; 1983.

23. Soule T, Garcia-Pichel F, Stout V: Gene expression patterns associated with the biosynthesis of the sunscreen scytonemin in Nostoc punctiforme ATCC 29133 in response to UVA radiation. J Bacteriol 2009, 19 I(I 4):4639-4646.

24. Stingele F, Newell JW, Neeser JR: Unraveling the function of glycosyltransferases in Streptococcus thermophilus Sfi6. J Bacteriol 1999, I8I(20):6354-6360.

25. Ehling-Schulz M, Bilger W, Scherer S: UV-B-induced synthesis of photoprotective pigments and extracellular polysaccharides in the terrestrial cyanobacterium Nostoc commune. J Bacteriol 1997, I79(6): 1940-1945.

26. Sanchez-Ferrer A, Rodriguez-Lopez JN, Garcia-Canovas F, GarciaCarmona F: Tyrosinase: a comprehensive review of its mechanism. Biochim Biophys Acta 1995, I 247: I-II.

27. Hoch J: Two-component and phosphorelay signal transduction. Curr Opin Microbiol 2000, 3:165-170.

28. Stock AM, Robinson VL, Goudreau PN: Two-component signal transduction. Annu Rev Biochem 2000, 69:183-215.

29. Hefti MH, Francoijs KJ, Devries SC, Dixon R, Vervoort J: The PAS fold. Eur J Biochem 2004, 27 I: I I 98-I 208.

30. Ashby MK, Houmard J: Cyanobacterial two-component proteins: structure, diversity, distribution, and evolution. Microbiol Mol Biol Rev 2006, 70:472-509.

31. Tobes R, Ramos JL: AraC-XyIS database: a family of positive transcriptional regulators in bacteria. Nucleic Acids Res 2002, 30(I):3।8-32I.

32. Castenholz RW: Cyanobacteria. In Bergey's Manual of Systematic Bacteriology Volume I. 2nd edition. Edited by: Boone D, Castenholz RW. New York: Springer; 2001:474-487.

33. Kobayashi A, Kajiyama SI, Inawaka K, Kanzaki H, Kawazu K: Nostodione A, a novel mitotic spindle poison from a blue-green alga Nostoc commune. Z Naturforsch 1994, 49:464-470.

34. Ploutno A, Carmeli S: Prenostodione, a novel UV-absorbing metabolite from a natural bloom of the cyanobacterium Nostoc species. J Nat Prod 200I, 64:544-545.

35. Tabata N, Sunazuka T, Tomoda H, Nagamitsu T, Iwai Y, Omura S: Diolmycins, new anticoccidial agents produced by Streptomyces sp. J Antibiot 1993, 46(5):762-769.

36. Christie PJ, Atmakuri K, Krishnamoorthy V, Jakubowski S, Cascales E: Biogenesis, architecture, and function of bacterial type IV secretion systems. Annu Rev Microbiol 2005, 59:45I-485. 
37. Stevenson CS, Capper EA, Roshak AK, Marquez B, Grace K, Gerwick WH, Jacobs RS, Marshall LA: Scytonemin, a marine natural product inhibitor of kinases key in hyperproliferative inflammatory diseases. Inflammation Res 2002, 5 I: | | 2-II4.

38. Stevenson CS, Capper EA, Roshak AK, Marquez B, Eichman C, Jackson JR, Mattern M, Gerwick WH, Jacobs RS, Marshall LA: The identification and characterization of the marine natural product scytonemin as a novel antiproliferative pharmacophore. J Pharmacol Exp Ther 2002, 303(2):858-866.

39. Allen M, Arnon DI: Studies on nitrogen-fixing blue-green algae. I. Growth and nitrogen-fixation by Anabaena cylindrica. Plant Physiol 1955, 30:366-372.

40. Waterbury JB, Stanier RY: Isolation and growth of cyanobacteria from marine and hypersaline environments. In The Prokaryotes Volume I. Edited by: Starr M. Berlin, Heidelberg, New York: Springer-Verlag; 1981:247-256.

41. Rippka R, Deruelles J, Waterbury JB, Herdman M, Stanier RY: Generic assignments, strain histories, and properties of pure cultures of cyanobacteria. J Gen Microbiol I979, I I I: I-6I.

42. Countway P, Gast R, Savai P, Caron D: Protistan diversity estimates based on I8S rDNA from seawater incubations in the western north Atlantic. J Euk Microbiol 2005, 52(2):95-106.

Publish with Bio Med Central and every scientist can read your work free of charge

"BioMed Central will be the most significant development for disseminating the results of biomedical research in our lifetime. "

Sir Paul Nurse, Cancer Research UK

Your research papers will be:

- available free of charge to the entire biomedical community

- peer reviewed and published immediately upon acceptance

- cited in PubMed and archived on PubMed Central

- yours - you keep the copyright

Submit your manuscript here:

http://www.biomedcentral.com/info/publishing_adv.asp
BiolMedcentral 